\title{
Vasospasm or Myocarditis: Cardiac Magnetic Resonance Plays a Decisive Role
}

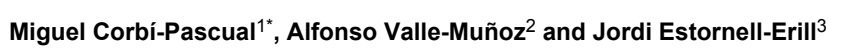

${ }^{1}$ Department of Cardiology, Hospital General de Albacete, Spain

${ }^{2}$ Department of Cardiology, Hospital de Denia, Spain

${ }^{3}$ ERESA, Unidad de TACy RMN Cardiaca, Valencia, Spain

*Corresponding author: Miguel Corbí Pascual, Avd. Hispanidad, 25 2-503804 Alcoy (Alicante), Spain, Tel: 0034-657370091; E-mail: miguelcorbi@hotmail.com

Received date: November 14, 2014; Accepted date: November 15, 2014; Published date: November 27, 2014

Copyright: (c) 2014 Corbí-Pascual M, et al. This is an open-access article distributed under the terms of the Creative Commons Attribution License, which permits unrestricted use, distribution, and reproduction in any medium, provided the original author and source are credited.

\section{Description}

A previously healthy 36-year-old male was admitted with acute onset chest pain and elevated levels of cardiac enzymes (CK/MB 248/25ng/dl and TnI 7.16ng/dl). He denied use of cocaine or other illicit drugs. Clinical history was negative for recent flu-like syndrome. Physical examination is unremarkable. ECG showed Q wave in lead III with $\mathrm{T}$ wave inversion (Figure 1A). An echocardiogram showed a structurally normal heart with no wall motion abnormalities. He had the abrupt recurrence of severe chest discomfort 12 hours later associated with ST segment elevation in I and aVL (Figure 1B). Symptoms were relieved after administration of intravenous nitroglycerin and ECG changes suddenly resolved. Urgent coronary angiography revealed normal coronary arteries, despite slow flow in the left anterior descending artery. The patient was diagnosed with AMI type 2 secondary to probably coronary artery spasm. Cardiac enzymes peaked at CK/MB 2107/147 and TnI 71. On day 4 a cardiac magnetic resonance (CMR) showed normal first pass and areas of subepicardial contrast enhancement in all segments except in the lateral wall at basal level and septal midventricular consistent with extensive myocarditis (Figure 1C).

Serology studies were negative. After 7 days the patient was discharged and remains asymptomatic.

Coronary vasospasm during the initial phase of acute myocarditis, in which presentation mimicked myocardial infarction, is a rare association. The possible pathogenic mechanism that might be implicated are an altered neurohumoral tone, release of vasoactive substances from aggregating platelets or immunologically mediated coronary vasculitis. CMR is such a powerful technique in the diagnosis of myocarditis and plays a key role in the differential diagnosis in acute coronary syndrome with normal coronary arteries based on the pattern of enhancement.

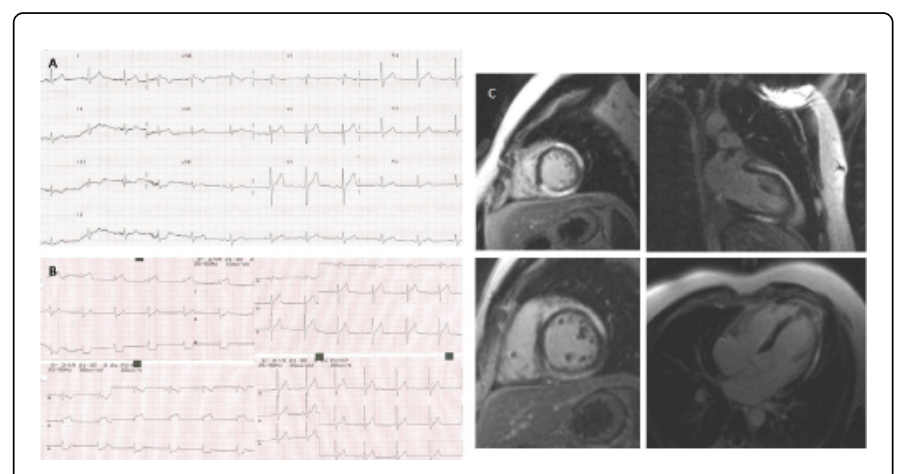

Figure 1: Vasospasm or Myocarditis: Cardiac Magnetic Resonance. 